\title{
Análise de desempenho e confiabilidade de reatores UASB em estações de tratamento de esgotos sanitários descentralizadas
}

\section{Performance and reliability analysis of UASB reactors in decentralized sewage treatment plants}

Data de entrada: 20/04/2020

Data de aprovação: $14 / 05 / 2020$

Ruam Magalhães da Silva² | Marcos Erick Rodrigues da Silva' | Paulo Igor Milen Firmino² | André Bezerra Dos Santos ${ }^{2 *}$

DOI: https://doi.org/10.36659/dae.2022.006

ORCID ID

Silva MER (D) https://orcid.org/0000-0002-6044-3159
Firmino PIM (D) https://orcid.org/0000-0002-3938-1264 Santos AB (iD) https://orcid.org/0000-0002-3395-8878

\section{Resumo}

O presente trabalho teve como objetivo realizar uma análise de desempenho e confiabilidade de reatores UASB em 18 estações de tratamento de esgotos sanitários descentralizadas em escala plena. A confiabilidade foi avaliada por meio da metodologia desenvolvida por Niku et al. (1979) para os parâmetros DQO e SST. A verificação da forma da distribuição de frequência desses parâmetros consistiu de testes de aderência às distribuições Normal, Lognormal, Gama e Exponencial, por meio dos testes Qui-quadrado e Kolmogorov-Smirnov. Verificou-se que os dados de DQO e SST efluentes aos reatores UASB seguiu uma distribuição lognormal. De uma forma geral, o desempenho dos reatores no atendimento aos padrões de descarte para os parâmetros DQO e SST não foi satisfatório. Em uma análise conjunta dos coeficientes de confiabilidade, concentrações de projeto e percentuais esperados de atendimento constatou-se a pouca confiabilidade da maioria dos reatores em relação aos constituintes DQ e SST, sendo indicativo de sobrecargas orgânicas e hidráulicas, respectivamente.

Palavras-chave: Desempenho. Confiabilidade. UASB. Estação de tratamento de esgotos. Padrões de lançamento.

\section{Abstract}

The present work aimed to perform a performance and reliability analysis of UASB reactors in 18 full-scale decentralized sewage treatment plants. Reliability was assessed using the methodology developed by Niku et al. (1979) for the parameters COD and SST. The verification of the form of the frequency distribution of these parameters consisted of tests of adherence to the Normal, Lognormal, Gamma and Exponential distributions, through the Chisquare and Kolmogorov-Smirnov tests. It was found that the COD and TSS effluent concentration data of UASB reactors followed a lognormal distribution. In general, the performance of the reactors in meeting the discharge standards for the COD and SST parameters was not satisfactory. In an integrated analysis of the reliability coefficients, project concentrations and expected compliance percentages, it was found the low reliability of most reactors in relation to the COD and SST constituents, being indicative of organic and hydraulic overload, respectively. Keywords: Performance. Reliability. UASB. Wastewater treatment plant. Discharge standards.

\footnotetext{
${ }^{1}$ Instituto Federal de Educação, Ciência e Tecnologia do Ceará (IFCE) - Fortaleza - Ceará - Brasil.

2 Universidade Federal do Ceará - Fortaleza - Ceará - Brasil.

* Autor correspondente: andre23aufc.br.
} 


\section{INTRODUÇÃO}

O uso de reatores anaeróbios de alta taxa, UASB e suas variantes, em Estações de Tratamento de Esgotos (ETEs) sanitários expandiu-se significativamente no mundo nas últimas duas décadas (NOYOLA et al., 2012). Atualmente, o Brasil possui o maior parque de reatores UASB do mundo em termos de unidades instaladas com foco no tratamento de esgoto sanitário (CHERNICHARO et al., 2018). Um conjunto de fatores contribuiu para tal disseminação, como o clima quente do nosso país, o amadurecimento da tecnologia, especialmente na própria engenharia nacional, a possibilidade de recuperação de recursos (biogás, lodo), a menor demanda de área em relação à tecnologia de lagoas de estabilização, a qual ainda é a principal rota tecnológica em ETEs em operação no Brasil e na América Latina (NOYOLA et al., 2012).

Contudo, os menores custos de implantação, operação e manutenção, por exemplo, quando comparados com sistemas aeróbios, como os dos lodos ativados, são de fato, a maior razão de tal disseminação, e são extremamente importantes para países em desenvolvimento como o Brasil, onde os déficits de saneamento são tão elevados. Segundo o recente Diagnóstico Nacional dos Serviços de Água e Esgoto (BRASIL, 2016), apenas $40,8 \%$ da vazão de esgoto gerada no país é tratada. Vários tipos de pós-tratamento de efluentes de reatores UASB vêm sendo utilizados no Brasil como lagoas de polimento (especialmente para ETEs afastadas dos centros urbanos ou locais de elevada disponibilidade de área), filtros biológicos percoladores, filtros aerados, lodos ativados, entre outros (DOS SANTOS, 2019). No âmbito do tratamento de esgotos, é importante não somente que o processo seja eficiente, com valores de remoção compatíveis com àqueles imaginados na concepção da ETE, mas também que a mesma seja confiável.
O conceito de confiabilidade está presente em vários trabalhos na área de engenharia sanitária, e uma definição bastante aceita para o termo é que a confiabilidade de um sistema é a probabilidade de obter um desempenho adequado por, pelo menos, um período específico de tempo e sob determinadas condições (NIKU et al., 1979). Assim, em termos de desempenho de um sistema de tratamento de esgoto, a confiabilidade pode ser entendida como a porcentagem de tempo em que se consegue cumprir os padrões de lançamento de efluentes. Por conseguinte, um sistema será completamente confiável se não houver falha no desempenho, ou seja, se não houver violação dos limites preconizados pelas legislações ambientais ou pela meta definida pela operadora.

Por sua vez, devido às inúmeras incertezas subjacentes ao projeto (variações nas características do esgoto, por exemplo) e às condições reais de operação das ETEs, a probabilidade de falha no cumprimento dos padrões de descarte deve sempre ser considerada no projeto e na formulação de legislações (ALDERSON et al., 2015; TAHERIYOUN; MORADINEJAD, 2015).

Muitas vezes, eficiência e confiabilidade são confundidas. A eficiência de um processo é relativa a uma determinada remoção em relação à concentração afluente. Já a confiabilidade é relativa aos dados de concentração efluente em relação a um determinado valor pré-estabelecido. Em outras palavras, uma ETE pode ser eficiente em termos médios e não ser confiável (elevado desvio padrão das concentrações efluentes), ou ser bastante confiável (baixo desvio padrão das concentrações efluentes) e não ser eficiente (baixas remoções na ETE).

Apesar de ser um tema de extrema relevância, nenhuma das legislações ambientais atualmente vigentes no Brasil contempla tal análise, sendo os padrões de lançamento principalmente avaliados em termos de concentração de efluente 
ou mesmo carga poluidora (MORAIS e DOS SANTOS, 2019). Assim, como comentado por Owusu-Ansah et al. (2015), a análise de desempenho de ETEs sem a devida inclusão da quantificação da conformidade ou atendimento aos padrões, principalmente, subestima os valores de descarga de efluentes considerados aceitáveis.

Metcalf (2016) define a confiabilidade $\left(\mathrm{C}_{\mathrm{f}}\right.$ ) como sendo uma função da probabilidade de falhas $(\alpha)$, que pode ser expressa pela Eq. 1:

$C f=1-\alpha$

Onde:

$\mathrm{C}_{\mathrm{f}}$ : confiabilidade do sistema ou probabilidade do atendimento aos padrões

a: probabilidade de falha do sistema ou probabilidade da concentração efluente superar a concentração requerida

Para determinar uma concentração média, que garanta que a concentração do efluente estará abaixo de certo valor, com um determinado nível de confiabilidade, Niku et al. (1979) desenvolveram um método que relaciona a concentração média do constituinte (valor de projeto) com os valores limites a serem cumpridos, baseandose em análises probabilísticas. 0 que se busca é avaliar, com base nessa metodologia, o grau de confiabilidade que foi alcançado pelos sistemas experimentais utilizados e compará-los com os sistemas reais, definindo assim o limite ou o padrão de efluente que poderíamos garantir em cada processo. Dessa forma, torna-se essencial que os processos de tratamento de esgotos sejam avaliados em relação à confiabilidade, fornecendo informações que podem subsidiar a seleção de um determinado sistema de tratamento e permitindo a avaliação do desempenho real de uma ETE no que se refere ao atendimento aos re- quisitos legais estabelecidos ou às metas de eficiência definidas durante o projeto.

Além disso, a determinação da confiabilidade operacional permite uma reflexão sobre a metodologia usualmente utilizada para definição da eficiência de projeto das ETEs, a qual normalmente não considera a variabilidade inerente a cada processo de tratamento, que está diretamente associada à confiabilidade. A probabilidade de falha é extremamente sensível à função de distribuição da concentração efluente. Uma vez conhecida a distribuição dos dados efluentes, uma expressão pode ser utilizada para definir a fração do tempo em que uma dada concentração foi excedida no passado e, desta forma, predizer o comportamento futuro de uma ETE, desde que as variáveis do processo continuem as mesmas.

Uma série de estudos publicados sobre a distribuição de dados de concentração de constituintes oriundos de estações de tratamento de esgoto (a maioria deles considerando DBO e SST) reporta que a distribuição lognormal fornece o melhor ajuste aos valores de concentração efluente (OLIVEIRA, 2006; MONTEIRO, 2009; SILVEIRA, 2011; ALDERSON et al., 2015). Em geral, a verificação da forma da distribuição de frequência dos indicadores dos efluentes das ETEs é efetuada em duas etapas. A primeira consiste na realização de testes de aderência às distribuições normal, lognormal, gama e exponencial, por meio dos testes do Qui-quadrado ( $x 2$ ), Lilliefors e Kolmogorov-Smirnov. Em um segundo momento, pode-se realizar o teste gráfico "Probability Probability Plot” para verificação da distribuição teórica que melhor se ajusta aos dados amostrais. Dentre os softwares utilizados para execução dos testes, podem-se citar: Estatística 7.0 e Statgraphics Centurion XVI.

Oliveira (2006) destaca que é comum os testes de aderência citados apresentarem mais de uma distribuição candidata como representativa dos 
dados amostrais. No caso dos testes do X2, os resultados são muito influenciados pelo número de intervalos de classes adotados. Os testes Kolmogorov-Smirnov são pouco precisos, indicando a possibilidade de aceitação de diversas distribuições como representantes do comportamento dos dados. Os testes de Lilliefors, uma extensão do teste de Kolmogorov-Smirnov, são mais precisos do que o teste original, não aceitando tantas distribuições candidatas; contudo, ainda se mostraram menos precisos que os testes do $\mathrm{X} 2$.

Nos estudos realizados anteriormente, a definição de uma distribuição de probabilidade mais adequada ao conjunto de dados operacionais de sistemas de tratamento de esgotos parece não ter ligação com o tipo de processo empregado. Foi observado que a qualidade e a quantidade de dados disponibilizados parecem ter um impacto maior nos resultados obtidos do que o tipo de tratamento. Adicionalmente, os estudos anteriores muitas vezes não levaram em consideração ETEs com elevado grau de descentralização, assim como contemplaram estações localizadas no Sudeste do Brasil, onde as características de operação podem ser diferentes.

O presente trabalho teve como objetivo realizar uma análise de desempenho e confiabilidade de reatores UASB em 18 estações de tratamento de esgotos sanitários descentralizadas em escala plena. A confiabilidade foi avaliada por meio da metodologia desenvolvida por Niku et al. (1979) para os parâmetros DQ Q e SST. A verificação da forma da distribuição de frequência desses parâmetros consistiu de testes de aderência às distribuições Normal, Lognormal, Gama e Exponencial, por meio dos testes Qui-quadrado e Kolmogorov-Smirnov.

\section{MATERIAL E MÉTODOS}

\subsection{Levantamento e organização dos dados}

Foram selecionadas 18 estações de tratamento localizadas em Fortaleza que tratavam seus esgotos sanitários por meio de reatores UASB seguidos de uma etapa de desinfecção simples pelo uso de cloração com hipoclorito de cálcio. Não foram cedidos dados de concentrações afluentes às ETEs, o que impossibilitou o cálculo da eficiência de remoção dos reatores.

Os conjuntos de dados disponibilizados eram compostos, às vezes, por 16 parâmetros e, em outros casos, por apenas quatro $(\mathrm{pH}, \mathrm{DQO}, \mathrm{SST}$ e E. coli). Algumas ETEs apresentaram dados relativos a todos os meses de monitoramento, enquanto outras forneciam dois resultados de análises no período analisado. Essa intermitência nos monitoramentos esteve presente em grande parte das estações de tratamento avaliadas.

Segundo Oliveira (2006), tais problemas possivelmente são decorrentes de mudanças nos planos de monitoramento, em que usualmente se aumenta a frequência dos parâmetros analisados, ou são adicionados novos parâmetros. Entretanto, em alguns casos pode ocorrer uma redução na frequência de alguns parâmetros, motivada pela inclusão de novas ETEs no plano de monitoramento. Outro problema observado foi a ausência de algum parâmetro por um ou mais meses. Por isso, optou-se por trabalhar apenas com os parâmetros DQO e SST, pois foram os constituintes de maior relevância e com maior número de dados coletados no período avaliado.

\subsection{Remoção de outliers}

Preliminarmente à estatística descritiva do número de dados, foi necessário verificar se os parâmetros monitorados apresentavam valores extremos, ou outliers. Para a identificação e exclusão desses dados, utilizou-se um teste 
empírico utilizado por Oliveira (2006), Monteiro (2009) e Silveira (2011). O teste consiste na determinação de limites inferior e superior, sendo estes calculados utilizando o primeiro e o terceiro quartil da série de dados, denominados de quartil inferior $\left(\mathrm{Q}_{\text {inf }}\right)$ e superior $\left(\mathrm{Q}_{\text {sup }}\right)$, respectivamente, como mostram as Eq. 2 e 3.

$$
\begin{aligned}
& L_{\text {inf }}=Q_{\text {inf }}-1,5\left(Q_{\text {sup }}-Q_{\text {inf }}\right) \\
& L_{\text {sup }}=Q s_{\text {up }}+1,5\left(Q_{\text {sup }}-Q_{i n f}\right)
\end{aligned}
$$

Assim, para todas as estações foram calculados os limites de cada parâmetro analisado. Caso o valor observado ficasse abaixo do limite inferior ou acima do limite superior, ele era considerado um outlier e, portanto, eliminado do conjunto de dados analisados.

\subsection{Estatística descritiva dos dados}

Após a remoção dos outliers, foram calculadas a média aritmética, mediana, moda, desvio padrão, valores máximos e mínimos, quartis inferior e superior e percentis de $10 \%$ e $90 \%$ dos dados coletados de cada constituinte.

A fim de facilitar a compreensão e verificar se as diferenças observadas entre os dois parâmetros eram estatisticamente significativas, com o auxílio do programa Excel foram elaborados gráficos do tipo box-plot. Nesses gráficos, são plotados os valores máximo e mínimo, o valor referente à mediana, além do $10^{\circ} \mathrm{e} 90^{\circ}$ percentil e do $1^{\circ} \mathrm{e} 3^{\circ}$ quartil dos dados coletados de cada parâmetro.

\subsection{Atendimento aos limites preconizados pela legislação}

Foi realizada uma análise dos dados efluentes dos parâmetros estudados por meio da estatística descritiva e as concentrações efluentes médias comparadas aos limites preconizados em legislações ambientais de alguns estados do Brasil, além de comparadas aos valores reportados na literatura especializada. Os padrões de lançamento estão descritos na Tabela 5. Conforme levantamento realizado por Morais e Dos Santos (2019), as faixas de valores reportados em legislações ambientais de alguns estados do Brasil para os parâmetros DQQO e SST foram de $100-250 \mathrm{mg} / \mathrm{L}$ e $60-150 \mathrm{mg} / \mathrm{L}$, respectivamente. Assim, para o presente trabalho, foram adotados os valores de 200 e 100 mg/L para a DQO e SST, respectivamente. É importante mencionar que a Resolução Conama $n^{\circ}$ 430/11 não traz valores de referência para esses parâmetros de lançamento.

Novamente, recursos, como gráficos box-plot, foram utilizados para melhorar a visualização e o entendimento da variabilidade dos dados efluentes das 18 estações de tratamento. Para quantificar a percentagem dos dados coletados que respeitaram os padrões de lançamento, foram utilizados gráficos de distribuição de probabilidade acumulada.

\subsection{Adoção de um modelo de distribuição de probabilidades}

A verificação da forma de distribuição de frequência dos indicadores consistiu de testes de aderência às distribuições Normal, Lognormal, Gama e Exponencial, por meio dos testes Quiquadrado e Kolmogorov-Smirnov, realizados com o auxílio dos programas Statgraphics Centurion XV e Statistica 8.0. Optou-se por utilizar sempre o número de intervalos de classe determinado pelo software Statgraphics, que resultava em valores bastante próximos da raiz quadrada do número de dados.

A partir da determinação do nível de significância a no valor de $5 \%$, para cada constituinte das estações foi obtida a probabilidade de aderência às quatro distribuições e, caso essa probabilidade 
fosse menor do que o nível de significância, podia-se afirmar ao nível de confiança de $95 \%$ que as frequências observadas diferiam da frequência esperada para a distribuição testada; caso contrário, a distribuição era elencada como candidata à distribuição mais representativa dos dados. Após a realização de todos os testes, aquelas distribuições que apresentaram as maiores probabilidades de aderência foram eleitas as mais representativas. Por vezes os dois testes apresentaram o mesmo modelo de distribuição como o mais indicado, e em outros casos os testes apontavam distribuições diferentes. Nessas situações, avaliou-se visualmente qual distribuição era a mais apropriada por meio do gráfico Probability - Probability Plot do programa Statistica.

\subsection{Cálculo da confiabilidade das estações}

Após a identificação do modelo distributivo e da estatística descritiva dos dados dos parâmetros avaliados com indicadores de desempenho das
ETEs, seguiu-se para a etapa de análise de confiabilidade dos sistemas de tratamento. Adotou-se a metodologia desenvolvida por Niku et al. (1979) e utilizada por em vários trabalhos (OLIVEIRA, 2006; MONTEIRO, 2009; SILVEIRA, 2011; ALDERSON et al., 2015), que permite o cálculo do coeficiente de confiabilidade (CDC) com o intuito de estimar a confiabilidade das estações de tratamento de esgoto, ou seja, estimar a fração do tempo em que a concentração efluente não excede o padrão de referência especificado. As Fig. 1 e 2 descrevem o procedimento de cálculo dos parâmetros para análise de confiabilidade dos sistemas.

O coeficiente de confiabilidade (CDC) é obtido a partir dos dados de monitoramento dos sistemas (média e desvio padrão) e da confiabilidade adotada a partir de uma probabilidade de falha aceitável. Além disso, a determinação da concentração média a ser adotada em projeto ou alcançada na operação, considerando a confiabilidade adotada, é obtida pelo produto do CDC de cada ETE pelo padrão adotado ou especificado na legislação (Fig. 1).

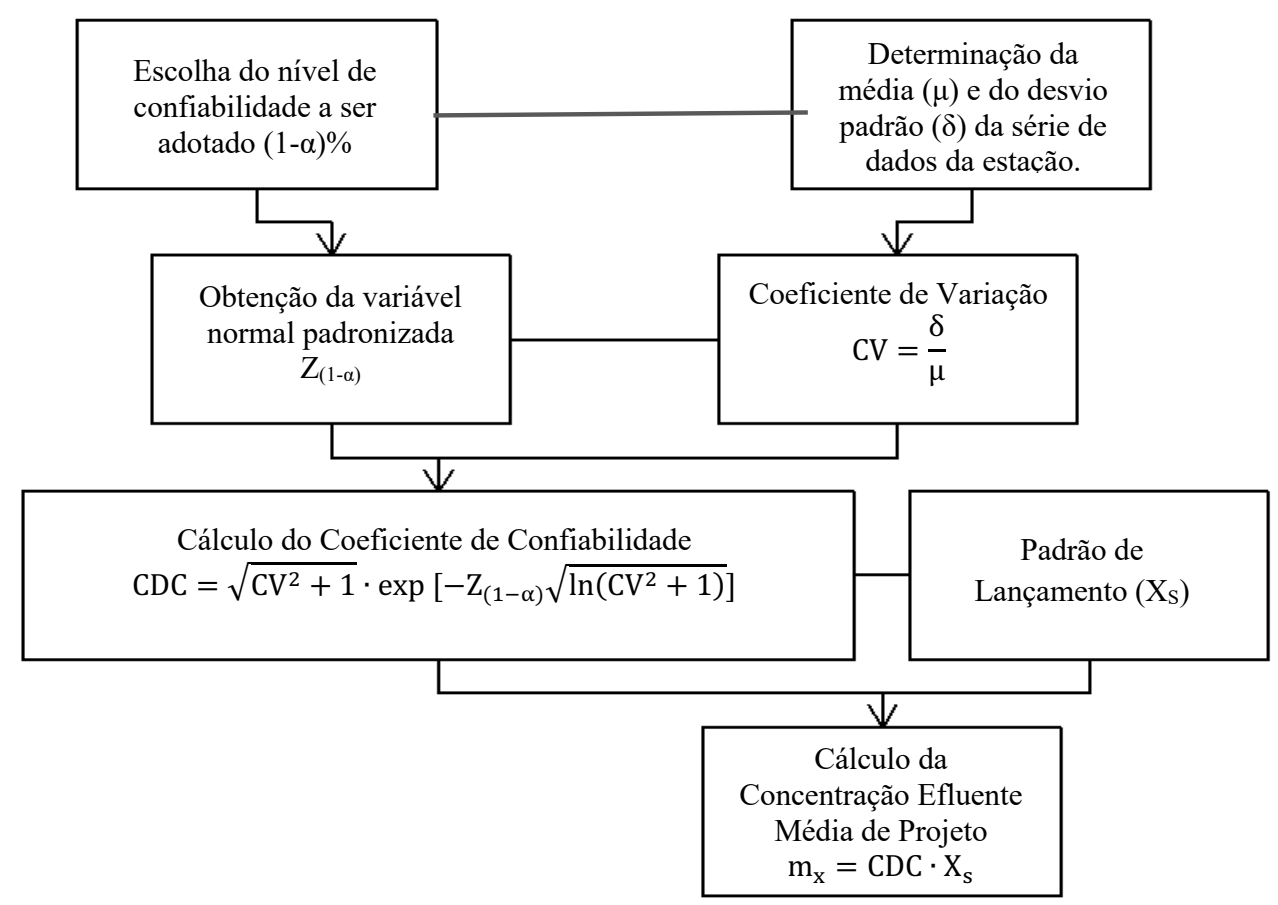

Figura 1 - Etapas para determinação do CDC e das concentrações de projeto. Fonte: Adaptado de Oliveira (2006). Obs. Os valores da variável normal padronizada $\left(Z_{(1-a)}\right)$ para os níveis de confiabilidade de 80,90 e $95 \%$ são $0,842,1,282$ e 1,645 , respectivamente. 
De posse dos valores das concentrações efluentes e dos coeficientes de variação de todas as ETEs estudadas, foi calculado o percentual esperado de atendimento às metas de lançamento adotadas. Para os valores de $Z_{(1-\alpha)}$ resultantes, foram obtidos os valores correspondentes à probabilidade cumulativa da distribuição normal padronizada (distribuição Z). Esses valores foram determinados por meio da função DIST.NORMP do Excel, e são equivalentes ao percentual de atendimento alcançado (Fig. 2).

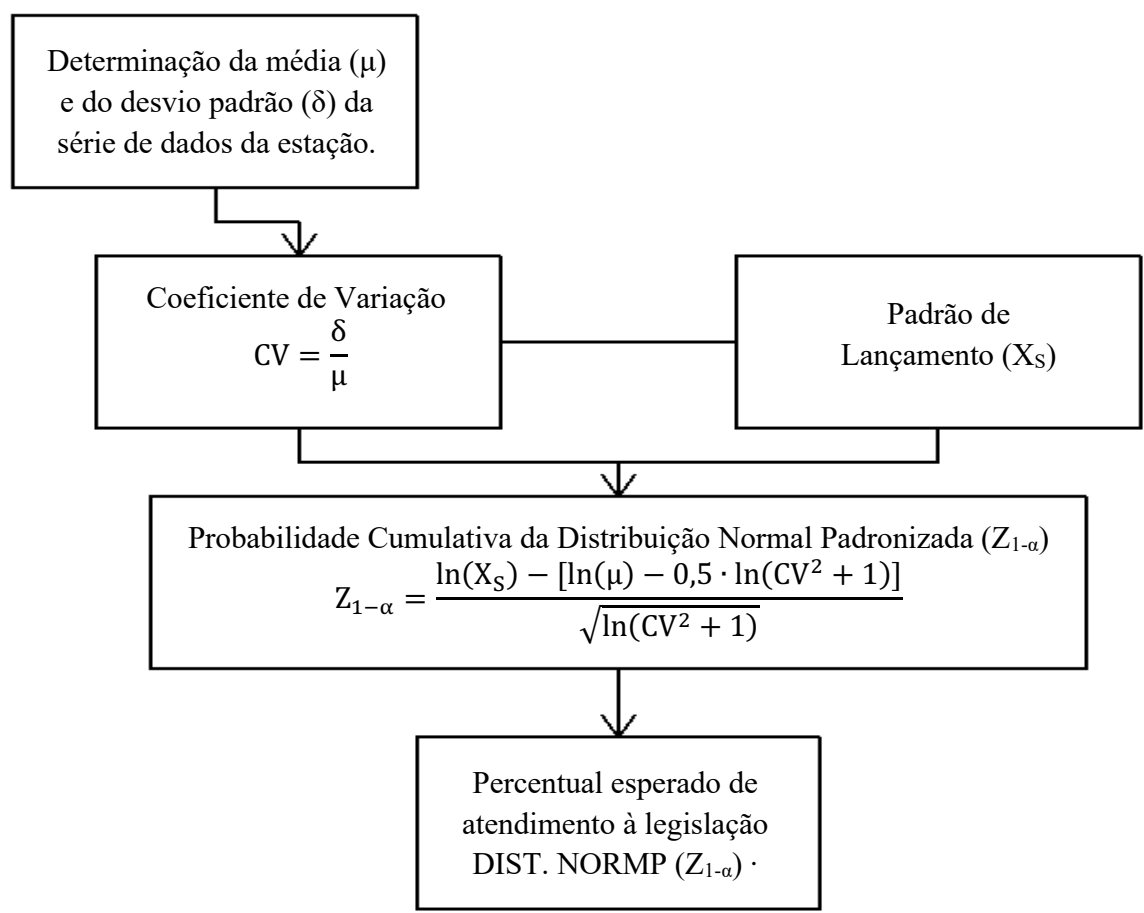

Figura 2 - Etapas para determinação do percentual esperado de atendimento à legislação. Fonte: Adaptado de Oliveira (2006).

Portanto, a análise da confiabilidade das ETEs foi realizada por meio dos coeficientes de confiabilidade para uma probabilidade de falha de $5 \%$, das concentrações de projeto ou metas operacionais e por meio dos percentuais esperados de atendimento aos padrões de lançamento ou confiabilidade esperada.

\section{RESULTADOS E DISCUSSÃO}

\subsection{Estatística descritiva referente às concentrações efluentes das ETEs}

Após a retirada dos outliers, foi feita a análise das concentrações efluentes dos parâmetros DQQ e
SST para cada estação de tratamento por meio da estatística descritiva. As Fig. 3 e 4 mostram, respectivamente, o gráfico box-plot das concentrações efluentes de DQO e SST para todas as estações de tratamento. É possível perceber a variabilidade dos dados de cada estação de tratamento pelas diferenças entre os valores máximos e mínimos (amplitude total) ou mesmo pelo intervalo interquartílico $\left(\mathrm{Q}_{3}-\mathrm{Q}_{2}\right)$ indicado pelo tamanho da caixa do box-plot.

Em geral, para os dois parâmetros avaliados, percebe-se que há uma forte relação entre a estabilidade das ETEs e a qualidade dos seus efluentes, ou seja, os sistemas que apresen- 
taram efluentes com menores concentrações de DQO e SST tiveram, também, menor variabilidade, portanto, eram mais estáveis. Dessa forma, como todos os sistemas de tratamento estudados operam prioritariamente com esgoto doméstico, pode-se inferir, de forma incipiente, uma tendência de que os sistemas mais eficientes sejam mais estáveis.

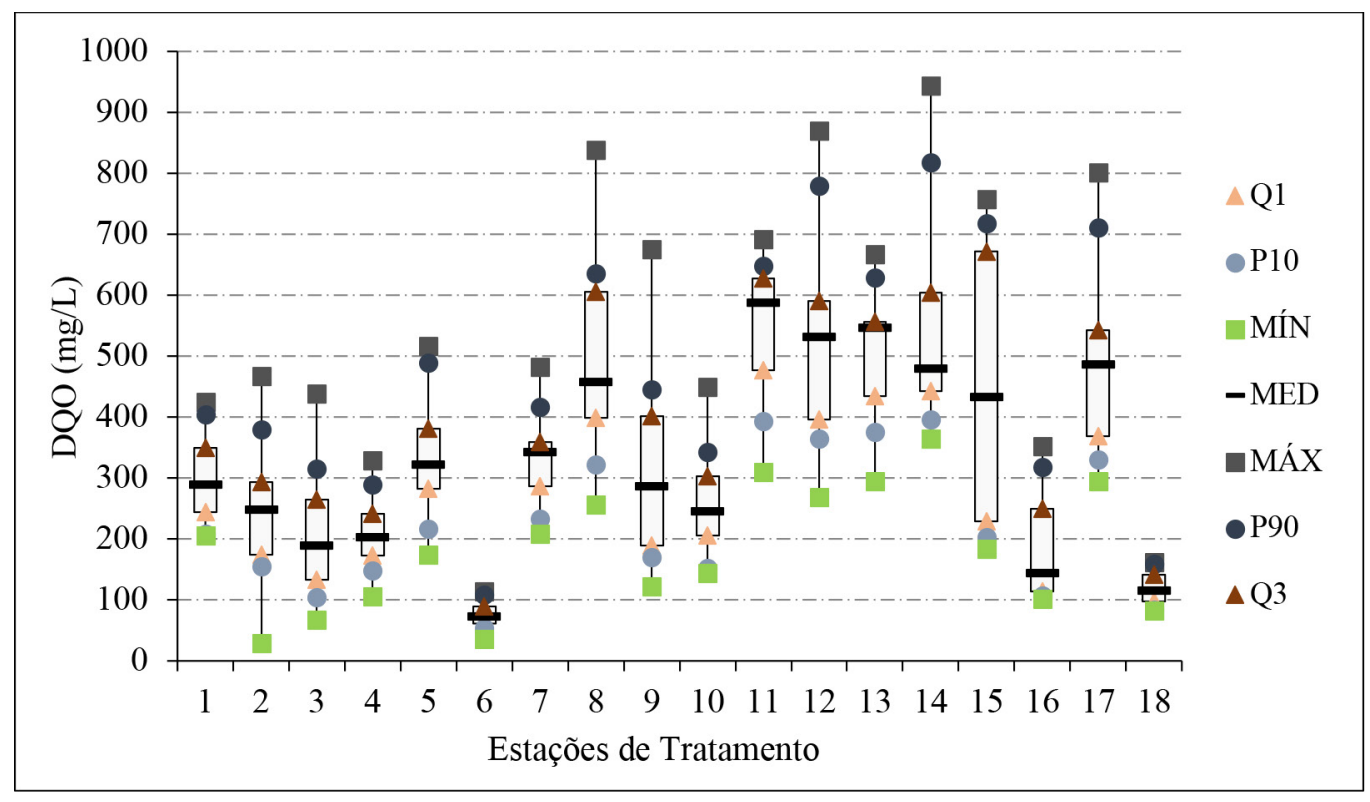

Figura 3 - Box-plot das concentrações efluentes de DQQO.

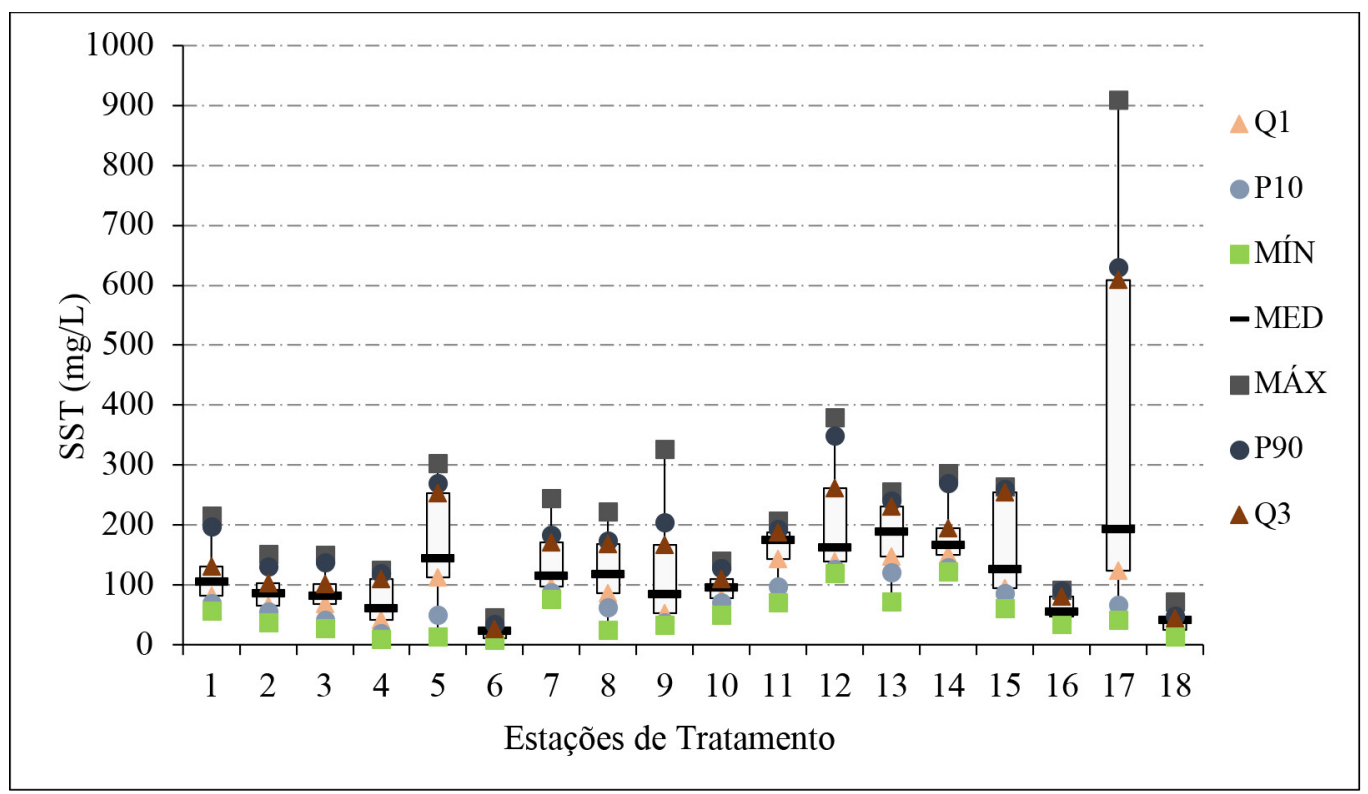

Figura 4 - Box-plot das concentrações efluentes de SST. 
Os valores das concentrações efluentes de DQQ encontrados variaram entre 29 e $944 \mathrm{mg} / \mathrm{L}$ com média de $341 \mathrm{mg} / \mathrm{L}$, entre todas as ETEs avaliadas. Segundo Von Sperling (2005), os valores usuais de DQO para efluentes de reatores UASB estão compreendidos entre 180 e 270 mg/L. Oliveira (2006), avaliando o desempenho de 166 ETEs em São Paulo e Minas Gerais, obteve concentrações de DQO para efluentes de reatores UASB com pós-tratamento (filtro aerado, filtro anaeróbio, lagoa facultativa, lagoa de polimento, entre outras) entre 61 e $219 \mathrm{mg} / \mathrm{L}$ e média de $141 \mathrm{mg} / \mathrm{L}$. A mesma autora obteve ainda para reatores UASB sem nenhum pós-tratamento, valores entre 344 e $147 \mathrm{mg} / \mathrm{L}$ e média de 251 $\mathrm{mg} / \mathrm{L}$. Tal diferença na qualidade do efluente tratado pode ser tanto decorrente de aspectos operacionais como relativos ao nível de descentralização da ETE, em que normalmente ETEs mais próximas à origem dos esgotos domésticos tendem a ter esgotos mais concentrados (DOS SANTOS, 2019).

Os valores das concentrações efluentes de SST variaram entre 9 e $910 \mathrm{mg} / \mathrm{L}$ com média de 131 $\mathrm{mg} / \mathrm{L}$. Segundo Von Sperling (2005), os valores usuais de SST para efluentes de reatores UASB encontram-se compreendidos entre 60 e 100 $\mathrm{mg} / \mathrm{L}$. Oliveira (2006) obteve concentrações de SST para efluentes de reatores UASB com póstratamento (filtro aerado, filtro anaeróbio, lagoa facultativa, lagoa de polimento, entre outras) entre 17 e $85 \mathrm{mg} / \mathrm{L}$ e média de $51 \mathrm{mg} / \mathrm{L}$. A mesma autora obteve ainda, para reatores UASB sem nenhum pós-tratamento, valores entre 49 e 137 e média de 85 mg/L. Monteiro (2009) obteve concentração média efluente de $150,3 \mathrm{mg} / \mathrm{L}$ para reatores UASB seguidos de cloração e média de $122,7 \mathrm{mg} / \mathrm{L}$ para reatores operando sem pós-tratamento.

Portanto, a partir da análise da qualidade do efluente das ETEs estudadas, constata-se gran- de variabilidade entre as estações, mesmo constituídas da mesma tecnologia de tratamento, podendo ser causada por falta de manutenção ou alteração nas características do afluente, apontado para a importância de um bom gerenciamento e operação dos sistemas.

\subsection{Adoção de um modelo de distribuição de probabilidades}

Com o auxílio dos softwares Statistica 8.0 e Statgraphics Centurion XV, foram aplicados os testes de aderência Kolmogorov-Smirnov e Qui-quadrado dos dados efluentes das ETEs em estudo para analisar os ajustes às distribuições Normal (N), Lognormal (LogN), Gama (G) e Exponencial (Exp). Os testes mostraram que na maioria dos casos as distribuições Gama, Normal e Lognormal podiam ser adotadas como representantes dos dados e que poucas estações de tratamento conseguiram ajuste de dados à distribuição Exponencial (dados não apresentados). Os casos em que não houve aderência a nenhuma das distribuições, assim como aderências a todas as distribuições, ocorreram em número reduzido. Com o auxílio de ferramentas do programa Statgraphics Centurion XV, determinou-se a distribuição que mais se ajustava à série de dados dentre aquelas já previamente selecionadas. Por vezes os testes Qui-quadrado e Kolmogorov-Smirnov apresentaram o mesmo modelo de distribuição como o mais representativo, e em outros casos os testes apontavam distribuições diferentes. Nessas situações, avaliou-se visualmente qual distribuição era a mais indicada por meio do gráfico Probability - Probability Plot do programa Statistica 8.0. Para cada constituinte, foi verificado o percentual de ajuste a cada distribuição e aquelas que apresentaram maior percentual foram adotadas como representantes do comportamento dos dados, como mostra a Tabela 1. 
Tabela 1 - Resultados percentuais e distribuição adotada para os constituintes analisados.

\begin{tabular}{|c|c|c|c|c|}
\hline \multirow{2}{*}{ Constituintes } & \multicolumn{3}{|c|}{$\begin{array}{c}\text { Distribuições selecionadas como representante do } \\
\text { comportamento dos dados (\% de ETEs) }\end{array}$} & Distribuição Adotada \\
\cline { 2 - 5 } & Normal & Lognormal & Outra & Lognormal \\
\hline DQO & 26 & 58 & 16 & Lognormal \\
\hline SST & 32 & 58 & 11 & L \\
\hline
\end{tabular}

Percebe-se que a distribuição lognormal pode ser adotada como representante do comportamento de todos os parâmetros. Embora não tenha sido a distribuição que melhor se ajustava aos dados em todos os casos, ela também não foi rejeitada. Logo, é possível utilizar a metodologia descrita por Niku et al. (1979) para o cálculo do coeficiente de confiabilidade (CDC) das estações de tratamento.

Resultados semelhantes foram obtidos por diversos autores que estudaram o comportamento de dados oriundos de estações de tratamento de esgotos (NIKU et al., 1979; BERTHOUEX; HUNTER, 1981, 1983; REDDA, 2013). Monteiro (2009) e Silveira (2011), analisando a confiabilidade de ETEs em Fortaleza e na sua Região Metropolitana, assim como Oliveira (2006), analisando o desempenho e a confiabilidade de 166 ETEs em São Paulo e Minas Gerais, também encontraram a distribuição lognormal como representante dos parâmetros para diversos processos de tratamento estudados.

\subsection{Cálculo do coeficiente de confiabilidade}

Como comentado no item 3.2, os resultados da caracterização das distribuições de probabilidade mostraram que a distribuição lognormal forneceu um bom ajuste aos dados dos constituintes DQO e SST, o que permitiu o emprego da metodologia desenvolvida e descrita por Niku et al. (1979) para a determinação dos coeficientes de confiabilidade a partir dos coeficientes de variação.

A partir da expressão mostrada na Fig. 1 para o cálculo do CDC, pode-se fazer uma análise de sensibilidade do CDC com o CV e nível de confiabilidade adotada para o sistema (Tabela 2 ).

Tabela 2 - Valores de CDC para diversos valores de CV e níveis de confiabilidade.

\begin{tabular}{|c|c|c|c|c|c|c|c|c|c|c|c|c|c|}
\hline $\begin{array}{c}\text { Niveis de } \\
\text { Confiabilidade }\end{array}$ & $\mathbf{0}$ & $\mathbf{0 , 2}$ & $\mathbf{0 , 4}$ & $\mathbf{0 , 6}$ & $\mathbf{0 , 8}$ & $\mathbf{1}$ & $\mathbf{1 , 2}$ & $\mathbf{1 , 4}$ & $\mathbf{1 , 6}$ & $\mathbf{1 , 8}$ & $\mathbf{2}$ & $\mathbf{2 , 5}$ & $\mathbf{3}$ \\
\hline $\mathbf{8 0} \%$ & 1 & 0,86 & 0,78 & 0,73 & 0,71 & 0,7 & 0,71 & 0,72 & 0,73 & 0,75 & 0,77 & 0,82 & 0,88 \\
\hline $90 \%$ & 1 & 0,79 & 0,66 & 0,57 & 0,52 & 0,49 & 0,47 & 0,45 & 0,44 & 0,44 & 0,44 & 0,44 & 0,45 \\
\hline $95 \%$ & 1 & 0,74 & 0,57 & 0,47 & 0,4 & 0,36 & 0,33 & 0,31 & 0,30 & 0,29 & 0,28 & 0,27 & 0,26 \\
\hline
\end{tabular}

Percebe-se que, para o mesmo nível de confiabilidade, os valores de CDC diminuem com o aumento dos valores de CV. Por exemplo, para um nível de confiabilidade de $90 \%$, uma ETE terá um CDC igual a 0,57 para o CV de 0,6 e um CDC de 0,52 quando o CV for igual a 0,8. Outro aspecto relevante diz respeito aos valores de CDC obtidos para os níveis de confiabi- lidade diferentes (80, 90 e 95\%). Observa-se que, para um valor de CV acima de 1,0 (para o nível de $80 \%$ ), maior que 2,0 (para o nível de $90 \%$ ), e acima de 4,0 (para o nível de $95 \%$ ), os CDCs obtidos se elevam, o que não apresenta significado físico, visto que não faria sentido a confiabilidade aumentar com a variabilidade dos dados. 
Portanto, os valores dos CDCs para os constituintes de interesse, considerando os coeficientes de variação (CV) efetivamente apresentados pelas ETEs em operação, foram calculados para um nível de confiabilidade de $95 \%$ (probabilidade de falha de $5 \%$ ). 0 valor médio obtido para CDC do parâmetro DQQ foi de 0,630, que difere bastante das médias 0,490 e 0,530, obtidas por Oliveira e von Sperling (2008) para reatores UASB sem e com pós-tratamento (filtro aerado, filtro anaeróbio, lagoa facultativa, lagoa de polimento, entre outras), respectivamente. Entretanto, o valor encontrado neste estudo se aproxima do valor de 0,580 obtido por Monteiro (2009) para a mesma tecnologia de tratamento, os reatores UASB seguidos de cloração, localizados em Fortaleza e na sua região metropolitana.

Das estações em estudo, considerado agora o parâmetro SST, o valor calculado para o CDC médio do parâmetro SST foi de 0,557 , próximo do valor de 0,520 obtido por Monteiro (2009) para reatores UASB também seguidos de cloração, mas distante das médias obtidas por Oliveira e von Sperling (2008), no valor de 0,440 para reatores sem pós-tratamento e 0,430 para UASB seguidos de pós-tratamento. Nesse último trabalho, é interessante observar que o pós-tratamento diminui a confiabilidade da ETE, revelando problemas operacionais diversos.

Em resumo, os reatores UASB em estudo apresentaram coeficientes de confiabilidade maiores do que os reportados na literatura. Entretanto, deve-se ressaltar que baixos valores de $\mathrm{CV}$ e, consequentemente, altos valores de CDC, não implicam em bons desempenhos, mas apenas uma condição mais estável de operação. Pequenos valores de coeficientes de confiabilidade implicam em menores valores de concentrações efluentes de projeto necessárias para o cumprimento de padrões de lançamento, como será comentado mais detalhadamente a seguir.

\subsection{Cálculos das concentrações de projeto}

A Tabela 3 apresenta, para os parâmetros DQQO e SST, os valores de CDC para um nível de confiabilidade de $95 \%$, padrão de lançamento $\left(X_{s}\right)$ e concentrações de projeto $\left(m_{x}\right)$ necessárias ao alcance das metas de lançamento adotadas.

Tabela 3 - Concentrações de projeto necessárias ao alcance dos padrões de descarte.

\begin{tabular}{|c|c|c|c|c|c|c|c|c|c|c|}
\hline \multirow{2}{*}{ ETEs } & \multicolumn{5}{|c|}{ DQQO } & \multicolumn{5}{|c|}{ SST } \\
\hline & $\mu(\mathrm{mg} / \mathrm{L})$ & $X_{s}(\mathrm{mg} / \mathrm{L})$ & CDC & $m_{x}(m g / L)$ & $m_{x} / \mu$ & $\mu(\mathrm{mg} / \mathrm{L})$ & $X_{s}(\mathrm{mg} / \mathrm{L})$ & CDC & $m_{x}(m g / L)$ & $\mathrm{mx} / \mu$ \\
\hline Média & 341 & \multirow{4}{*}{200} & 0,630 & 126 & 0,50 & 128 & \multirow{4}{*}{100} & 0,557 & 56 & 0,63 \\
\hline Desv. Padrão & 153 & & 0,072 & 14 & 0,38 & 70 & & 0,082 & 8 & 0,52 \\
\hline Mínimo & 77 & & 0,502 & 100 & 0,23 & 23 & & 0,384 & 38 & 0,12 \\
\hline Máximo & 548 & & 0,733 & 147 & 1,72 & 317 & & 0,681 & 68 & 2,33 \\
\hline
\end{tabular}

Como exemplo de interpretação da Tabela 3, em termos médios de operação de reatores UASB no tratamento de esgotos sanitários foi obtido um CDC no valor de 0,630. Assim, o reator UASB teria que ser projetado para fornecer uma concentração efluente média máxima de 126 $\mathrm{mg} / \mathrm{L}$ para garantir que $95 \%$ dos resultados alcançassem a meta de $200 \mathrm{mg} / \mathrm{L}$. Outra forma de interpretação é que a operação da ETE deverá conduzir ao valor médio de $126 \mathrm{mg} / \mathrm{L}$ de DQO no efluente para que, com a variabilidade inerente àquela estação, $95 \%$ dos dados estejam abaixo de $200 \mathrm{mg} / \mathrm{L}$. Quanto menor a concentração de projeto $\left(m_{x}\right)$ em uma ETE, maior a probabilidade de falhas que podem ocorrer na mesma, ou seja, baixos valores de $m_{x}$ indicam baixa confiabilidade operacional e altos valores indicam condições mais estáveis de operação. 
Outro ponto relevante diz respeito às razões entre as concentrações de projeto e as concentrações reais observadas para as estações de tratamento, que levariam ao alcance das metas estabelecidas. Observa-se que, para uma razão igual a 1, a concentração efluente real observada para a ETE coincidiria com o valor de projeto necessário para garantir que $95 \%$ dos resultados atendessem às metas de lançamento adotadas. No entanto, em geral, foram observadas grandes diferenças entre as concentrações necessárias para atendimento das metas e aquelas efetivamente observadas, com a grande maioria das ETEs apresentando valores bem abaixo de 1,0, para todos os constituintes.

\subsection{Cálculo do percentual esperado de atendimento à legislação}

A análise isolada do CDC determina apenas o quanto uma ETE ou tecnologia de tratamen- to é operacionalmente estável, enquanto a avaliação conjunta do coeficiente de confiabilidade $(C D C)$, concentração média efluente $(\mu)$, concentração de projeto $(m x)$ e percentual esperado de atendimento à meta para um determinado nível de confiabilidade possibilitam o conhecimento do desempenho das ETES de uma mesma modalidade de tratamento, bem como a comparação de modalidades de tratamento distintas.

Conhecidos os valores das concentrações efluentes e dos coeficientes de variação de todas as ETEs estudadas, foi calculado o percentual esperado de atendimento às metas de lançamento adotadas por meio da função "DISTNORMP" do Excel (Tabela 4), conforme descrito no item 2.6 da Metodologia.

Tabela 4 - Percentual esperado de atendimento à legislação.

\begin{tabular}{|c|c|c|c|c|c|c|c|c|c|c|}
\hline \multirow[b]{2}{*}{ ETEs } & \multicolumn{5}{|c|}{ DQQ } & \multicolumn{5}{|c|}{ SST } \\
\hline & $\mu(\mathrm{mg} / \mathrm{L})$ & $X_{s}(\mathrm{mg} / \mathrm{L})$ & CV & $Z_{(1-\alpha)}$ & $\begin{array}{c}\text { Atend. } \\
\text { Esperado } \\
\%\end{array}$ & $\mu(\mathrm{mg} / \mathrm{L})$ & $X_{s}(m g / L)$ & CV & $Z_{(1-\alpha)}$ & $\begin{array}{c}\text { Atend. } \\
\text { Esperado } \\
\%\end{array}$ \\
\hline 1 & 299 & 200 & 0,243 & $-1,565$ & $5,9 \%$ & 117 & 100 & 0,411 & $-0,196$ & $42,2 \%$ \\
\hline 2 & 248 & 200 & 0,413 & $-0,349$ & $36,4 \%$ & 89 & 100 & 0,362 & 0,496 & $69,0 \%$ \\
\hline 3 & 205 & 200 & 0,460 & 0,158 & $56,3 \%$ & 87 & 100 & 0,394 & 0,568 & $71,5 \%$ \\
\hline 4 & 212 & 200 & 0,281 & $-0,067$ & $47,3 \%$ & 69 & 100 & 0,573 & 0,971 & $83,4 \%$ \\
\hline 5 & 336 & 200 & 0,292 & $-1,667$ & $4,8 \%$ & 167 & 100 & 0,537 & $-0,764$ & $22,2 \%$ \\
\hline 6 & 77 & 200 & 0,284 & 3,591 & $100,0 \%$ & 23 & 100 & 0,479 & 3,504 & $100,0 \%$ \\
\hline 7 & 332 & 200 & 0,226 & $-2,166$ & $1,5 \%$ & 132 & 100 & 0,357 & $-0,629$ & $26,5 \%$ \\
\hline 8 & 489 & 200 & 0,309 & $-2,804$ & $0,3 \%$ & 121 & 100 & 0,446 & $-0,225$ & $41,1 \%$ \\
\hline 9 & 304 & 200 & 0,464 & $-0,729$ & $23,3 \%$ & 111 & 100 & 0,747 & 0,183 & $57,3 \%$ \\
\hline 10 & 257 & 200 & 0,325 & $-0,632$ & $26,4 \%$ & 96 & 100 & 0,262 & 0,290 & $61,4 \%$ \\
\hline 11 & 544 & 200 & 0,208 & $-4,754$ & $0,0 \%$ & 160 & 100 & 0,257 & $-1,733$ & $4,2 \%$ \\
\hline 12 & 531 & 200 & 0,310 & $-3,074$ & $0,1 \%$ & 203 & 100 & 0,438 & $-1,476$ & $7,0 \%$ \\
\hline 13 & 508 & 200 & 0,203 & $-4,545$ & $0,0 \%$ & 183 & 100 & 0,287 & $-2,017$ & $2,2 \%$ \\
\hline 14 & 548 & 200 & 0,325 & $-3,025$ & $0,1 \%$ & 181 & 100 & 0,292 & $-1,935$ & $2,7 \%$ \\
\hline 15 & 447 & 200 & 0,483 & $-1,526$ & $6,4 \%$ & 159 & 100 & 0,486 & $-0,777$ & $21,8 \%$ \\
\hline 16 & 189 & 200 & 0,524 & 0,356 & $63,9 \%$ & 62 & 100 & 0,385 & 1,486 & $93,1 \%$ \\
\hline 17 & 492 & 200 & 0,311 & $-2,810$ & $0,2 \%$ & 317 & 100 & 0,876 & $-1,151$ & $12,5 \%$ \\
\hline 18 & 119 & 200 & 0,239 & 2,328 & $99,0 \%$ & 37 & 100 & 0,471 & 2,445 & $99,3 \%$ \\
\hline
\end{tabular}

Boa parte das estações de tratamento apresentaram baixíssimos percentuais esperados de atendimento, tanto em relação ao parâme- tro DQQO como a SST, e algumas ETEs chegaram a apresentar percentual nulo ou quase próximo a isso. Nessa lista estão inclusas as estações de 
tratamento que obtiveram os maiores valores de CDC, ou seja, mesmo com altos valores de coeficiente de confiabilidade, indicando estabilidade operacional, essas estações não cumpriram os padrões de descarte.

Outra análise pertinente dos dados diz respeito às diferenças entre percentuais de atendimento para estações com coeficientes de confiabilidade semelhantes. Como exemplo dessa situação, têm-se o caso das ETEs 5 e 6, que apesar de possuírem coeficientes de variação parecidos, e consequentemente CDCs semelhantes $(0,658$ e 0,650 , respectivamente), possuem percentuais esperados de atendimento à meta de DOQ bem diferentes ( $100 \%$ e $4,8 \%$, respectivamente). Isso ocorre devido à diferença existente entre as concentrações médias efluentes das duas ETEs (77 e $336 \mathrm{mg} / \mathrm{L}$, respectivamente).

Assim, conclui-se que aquelas ETEs que já produzem concentrações efluentes menores terão uma maior probabilidade e facilidade de alcançar a concentração média com a qual os efluentes devem ser lançados para que as metas estabelecidas para DQO e SST sejam atendidas. O cálculo do CV leva em consideração o desvio padrão e a média dos dados efluentes e o CDC calculado a partir do CV e do nível de confiabilidade adotado, nesse caso de $95 \%$. Já a concentração de projeto $\left(m_{x}\right)$ é calculada a partir do produto entre o CDC e a meta especificada pela legislação $\left(X_{S}\right)$. Dessa forma, quando valores de CDC de duas ETEs distintas são iguais, os valores de $m_{x}$ são semelhantes, ou seja, os valores de CV utilizados no cálculo do CDC são iguais. Portanto, como o percentual esperado de atendimento à meta é calculado utilizando o padrão estabelecido pela legislação, o CV e a média efluente dos dados, o que determinará o valor do percentual será a média.

\subsection{Testes para comprovação da validade do modelo de confiabilidade}

Para comprovar a validade das projeções de desempenho efetuadas com base nos coeficientes de confiabilidade, os percentuais de atendimento esperados foram comparados com os percentuais de atendimentos efetivamente obtidos pelas ETEs para os padrões de lançamento $\left(X_{S}\right)$ adotados, calculados e apresentados no item 2.4.

Tabela 5 - Resultados reais e esperados para atendimento às metas de lançamento.

\begin{tabular}{|c|c|c|c|c|c|c|}
\hline \multirow[b]{2}{*}{ ETEs } & \multicolumn{3}{|c|}{ DQQO } & \multicolumn{3}{|c|}{ SST } \\
\hline & $X_{\mathrm{s}}(\mathrm{mg} / \mathrm{L})$ & $\begin{array}{l}\text { Atendimento } \\
\text { Observado (\%) }\end{array}$ & $\begin{array}{c}\text { Atend. Esperado } \\
\%\end{array}$ & $X_{s}(m g / L)$ & $\begin{array}{l}\text { Atendimento } \\
\text { Observado (\%) }\end{array}$ & $\begin{array}{c}\text { Atend. Esperado } \\
\%\end{array}$ \\
\hline 1 & 200 & $0,0 \%$ & $5,9 \%$ & 100 & $0,0 \%$ & $42,2 \%$ \\
\hline 2 & 200 & $32,0 \%$ & $36,4 \%$ & 100 & $8,0 \%$ & $69,0 \%$ \\
\hline 3 & 200 & $53,0 \%$ & $56,3 \%$ & 100 & $13,0 \%$ & $71,5 \%$ \\
\hline 4 & 200 & $45,0 \%$ & $47,3 \%$ & 100 & $35,0 \%$ & $83,4 \%$ \\
\hline 5 & 200 & $6,0 \%$ & $4,8 \%$ & 100 & $10,0 \%$ & $22,2 \%$ \\
\hline 6 & 200 & $100,0 \%$ & $100,0 \%$ & 100 & $100,0 \%$ & $100,0 \%$ \\
\hline 7 & 200 & $0,0 \%$ & $1,5 \%$ & 100 & $0,0 \%$ & $26,5 \%$ \\
\hline 8 & 200 & $0,0 \%$ & $0,3 \%$ & 100 & $7,0 \%$ & $41,1 \%$ \\
\hline 9 & 200 & $31,0 \%$ & $23,3 \%$ & 100 & $28,0 \%$ & $57,3 \%$ \\
\hline 10 & 200 & $25,0 \%$ & $26,4 \%$ & 100 & $0,0 \%$ & $61,4 \%$ \\
\hline 11 & 200 & $0,0 \%$ & $0,0 \%$ & 100 & $0,0 \%$ & $4,2 \%$ \\
\hline 12 & 200 & $0,0 \%$ & $0,1 \%$ & 100 & $0,0 \%$ & $7,0 \%$ \\
\hline 13 & 200 & $0,0 \%$ & $0,0 \%$ & 100 & $0,0 \%$ & $2,2 \%$ \\
\hline 14 & 200 & $0,0 \%$ & $0,1 \%$ & 100 & $0,0 \%$ & $2,7 \%$ \\
\hline 15 & 200 & $7,0 \%$ & $6,4 \%$ & 100 & $0,0 \%$ & $21,8 \%$ \\
\hline 16 & 200 & $64 \%$ & $63,9 \%$ & 100 & $30,0 \%$ & $93,1 \%$ \\
\hline 17 & 200 & $0,0 \%$ & $0,2 \%$ & 100 & $3,0 \%$ & $12,5 \%$ \\
\hline 18 & 200 & $100 \%$ & $99,0 \%$ & 100 & $90,0 \%$ & $99,3 \%$ \\
\hline
\end{tabular}


Pela comparação dos percentuais, percebe-se que o comportamento esperado de desempenho está muito próximo do comportamento observado, considerando os dois parâmetros analisados. Isso viabiliza a utilização do modelo de confiabilidade desenvolvido por Niku et al. (1979), que efetivamente descreve o comportamento de dados que seguem uma distribuição lognormal de probabilidade.

\section{CONCLUSÃO}

Verificou-se que os dados de DQO e SST efluentes aos reatores UASB seguiu uma distribuição lognormal. De uma forma geral, o desempenho dos reatores no atendimento aos padrões de descarte para os parâmetros DQQ e SST não foi satisfatório. Em uma análise conjunta dos coeficientes de confiabilidade, concentrações de projeto e percentuais esperados de atendimento, constatou-se a pouca confiabilidade da maioria dos reatores em relação aos constituintes $\mathrm{DQO}$ e SST. De todo modo, verifica-se a grande importância da análise de confiabilidade com as análises de eficiência e de atendimento ao requisito legal para entender melhor determinadas rotas tecnológicas adotadas e quais aspectos técnicos e operacionais podem ser aperfeiçoados.

\section{CONTRIBUIÇÃO DOS AUTORES}

Conceitualização e Orientação: Santos AB; Investigação: Silva RM; Redação: Santos $A B$, Silva RM, Silva MER, Firmino PIM.

\section{REFERÊNCIAS}

ALDERSON, M. P.; DOS SANTOS A. B.; MOTA FILHO C. R. Reliability analysis of low-cost, full-scale domestic wastewater treatment plants for reuse in aquaculture and agriculture. Ecological Engineering, v. 82, p. 6-14, 2015. https://doi.org/10.1016/j.ecoleng.2015.04.081.
BERTHOUEX, P. M.; HUNTER, W. G. How to construct reference distributions to evaluate treatment plant effluent quality. Journal of Water Pollution Control Federation, v. 55, n. 12, 1983.

BERTHOUEX, P. M.; HUNTER, W.G. Simple statistics for interpreting environmental data. Journal of Water Pollution Control Federation, v. 53, n. 2, 1981

BRASIL, Conselho Nacional de Meio Ambiente. Resolução 430 de 13 de maio de 2011 - Dispõe sobre as condições e padrões de lançamento de efluentes, complementa e altera a Resolução no 357, de 17 de março de 2005. Ministério do Meio Ambiente. Brasília. 2011.

BRASIL, Ministério das Cidades. Secretaria Nacional de Saneamento Ambiental - SNSA. Sistema Nacional de Informações sobre Saneamento: Diagnóstico dos Serviços de Água e Esgotos - 2016. Brasília: SNSA/MCIDADES, p. 218, 2018.

CHERNICHARO, C. A. L.; BRESSANI-RIBEIRO, T.; PEGORINI, E. S.; POSSETTI, G. R. C.; MIKI, M. K.; SOUZA, S. N. Contribuição para a melhoria de projeto, construção e operação de reatores UASB aplicados ao tratamento de esgoto sanitário - Parte 1: Tópicos de Interesse. Revista DAE, v.66, n. 214, p.5-16, 2018. https://doi.org/ 10.4322/dae.2018.038

DOS SANTOS, A. B.; CHERNICHARO, C. A. L.; LAPOLLI, F. R.; VON SPERLING, M.; KATO, M. T.; PIVELLI, R. P.; BRESSANI-RIBEIRO, T. Tecnologias de tratamento de correntes de esgotos não segregadas aplicadas a empreendimentos habitacionais. In: DOS SANTOS, A. B. Organizador. Caracterização, tratamento e gerenciamento de subprodutos de correntes de esgotos segregadas e não segregadas em empreendimentos habitacionais. Fortaleza: Imprece, 2019. Cap. 4, 219-391.

METCALF, L.; EDDY, H. P. Tratamento de efluentes e recuperação de recursos. Porto Alegre: AMGH. p. 1980, 2016.

MONTEIRO, C. R. L. Análise da eficiência e confiabilidade em 56 estações de tratamento de esgotos localizadas na região metropolitana de Fortaleza. 2009. Dissertação (Mestrado em Saneamento Ambiental) - Centro de Tecnologia, Universidade Federal do Ceará, Fortaleza, 2006.

MORAIS N. W. S; DOS SANTOS A. B.; Análise dos padrões de lançamento de efluentes em corpos hídricos e de reúso de águas residuárias de diversos estados do Brasil. Revista DAE, n. 215, v. 67, p. 40-55, 2019. https://doi.org/10.4322/dae.2019.004

NIKU S, SCHROEDER E. D.; SAMANIEGO F. J. Performance of activated sludge process and reliability-based design. Journal Water Pollution Control Association, n.51, v.12, p.2841-2857, 1979.

NOYOLA, A.; PADILLA-RIVERA, A.; MORGAN-SAGASTUME, J.; GUERECA, L. P.; HERNÁNDEZ-PADILLA, F. Typology of municipal wastewater treatment technologies in Latin America. CleanSoil, Air, Water v.40, p.926-932, 2012. https://doi.org/10.1002/ clen.201100707 
OLIVEIRA S. C.; VON SPERLING M. Reliability analysis of wastewater treatment plants. J. Water Research. v. 42, p. 1182-1194, 2008. https://doi.org/10.1016/j.watres.2007.09.001

OLIVEIRA, S. M. A. C. O. Análise de desempenho e confiabilidade de estações de tratamento de esgotos. Tese (Doutorado em Saneamento Ambiental) - Escola de Engenharia, Universidade Federal de Minas Gerais, Belo Horizonte, 2006.

OWUSU-ANSAH E. D. G. J.; SAMPSON A.; AMPONSAH S. K.; ABAIDOO, R.C.; HALD, T. Performance, compliance and reliability of Waste stabilization pond: Effluent discharge quality and environmental protection agency standards in Ghana. Research Journal of Applied Science, Engineering and Technology, n.10, v.11, p.1293-1302, 2015. http://dx.doi.org/10.19026/rjaset.10.1825.
SILVEIRA, A. G. M. Análise de eficiência e confiabilidade em sistemas de baixo custo de tratamento de esgotos do tipo lagoas de estabilização. Dissertação (Mestrado em Saneamento Ambiental) - Centro de Tecnologia, Universidade Federal do Ceará, Fortaleza, 2011.

TAHERIYOUN, M.; MORADINEJAD, S. Reliability analysis of a wastewater treatment plant using fault tree analysis and Monte Carlo simulation. Environmental Monitoring and Assessment, v. 187, 2015. https://doi.org/10.1007/s10661-014-4186-7

VON SPERLING, M. Introdução à qualidade das águas e ao tratamento de esgotos. 3 ed. Belo Horizonte: UFMG, 2005. (Princípios do tratamento biológico de águas residuárias, v. 1). 